PROCEEDINGS OF THE

AMERICAN MATHEMATICAL SOCIETY

Volume 37, Number 1, January 1973

\title{
NILPOTENT ELEMENTS IN BANACH ALGEBRAS
}

\author{
H. BEHNCKE
}

\begin{abstract}
Let $\mathfrak{U}$ be an $A^{*}$-algebra such that any maximal abelian *-subalgebra is regular and such that any quasinilpotent element $x$ in $\mathfrak{U}$ satisfies $x^{N}=0$, with $N<\infty$. Then any irreducible Hilbert space *-representation is at most $N$-dimensional. If $\mathfrak{U}$ is a $C^{*}$-algebra, $\mathfrak{U}$ possesses transcendental quasinilpotent elements if there exists a $\pi \in \hat{\mathfrak{U}}$ with $\operatorname{dim} \pi=\infty$.
\end{abstract}

This note is a continuation of [1]. We want to establish an intimate connection between the degree of noncommutativity of a Banach algebra $\mathfrak{U}$ and the existence of nilpotent elements in $\mathfrak{U}$. The proofs are straightforward extensions of the matrix situation and of methods used in [1].

Throughout all Banach algebras are complex Banach algebras with norm || , continuous involution $*$ and an auxiliary norm [3] ||$_{0}$ which satisfies $\left|x x^{*}\right|_{0}=|x|_{0}^{2}$. Such Banach algebras are called $A^{*}$-algebras [3]. In $A^{*}$-algebras maximal abelian *-subalgebras are necessarily semisimple. We shall further assume that all Banach algebras have a unit, because the adjunction of a unit does not affect the proofs nor the results. A representation $\pi$ always denotes a *-representation on a Hilbert space $H_{\pi}$. We say that $x \in \mathfrak{U}$ is $n$-nilpotent, with $n=\infty, 1,2, \cdots$, if $x$ is quasinilpotent and $x^{n}=0$ but $x^{n-1} \neq 0$.

We begin with a simple lemma, which is probably known.

LEMMA. Let $\mathfrak{I}$ be an $A^{*}$-algebra such that any $x=x^{*} \in \mathfrak{U}$ has a finite spectrum with at most $n$ points; then $\operatorname{dim} \mathfrak{U} \leqq n^{2}$.

Proof. Let $\mathfrak{B}$ be a maximal abelian (semisimple)*-subalgebra of $\mathfrak{A}$. Then the character space $\hat{\mathfrak{B}}$ of $\mathfrak{B}$ has at most $n$ elements. Hence any $x \in \mathfrak{B}$ has the form $x=\sum_{i \leqq n} \lambda_{i} e_{i}$, where the $e_{i}$ are the minimal idempotents of $\mathfrak{B}$. The $e_{i}$ are selfadjoint and it is easy to see that $\operatorname{dim} e_{i} \mathfrak{A} e_{j} \leqq 1$.

THEOREM 1. (i) Let $\mathfrak{U}$ be an $A^{*}$-algebra such that any maximal abelian *-subalgebra is regular and such that any quasinilpotent element $x$ in $\mathfrak{U}$ satisfies $x^{N}=0$, then any irreducible representation $\pi$ of $\mathfrak{A}$ is at most $N$ dimensional.

Received by the editors February 10, 1972.

AMS (MOS) subject classifications (1969). Primary 4660, 4665.

Key words and phrases. Nilpotent elements, Banach ${ }^{*}$-algebras, $C^{*}$-algebras.

(c) American Mathematical Society 1973 
(ii) Let $\mathfrak{U}$ be an $A^{*}$-algebra such that any maximal abelian *-subalgebra of $\mathfrak{U}$ is regular and assume $\mathfrak{U}$ has an irreducible representation $\pi$ of dimension $1<N<\infty$, then $\mathfrak{U}$ has an $N$-nilpotent element.

(iii) Let $\mathfrak{U}$ be an $A^{*}$-algebra such that any irreducible representation of $\mathfrak{U}$ is at most $N$-dimensional; then any quasinilpotent element $x$ in $\mathfrak{A}$ satisfies $x^{N}=0$.

Proof. (i) Let $\pi$ be an irreducible representation of $\mathfrak{A}$ and let $\mathfrak{B}$ be a maximal abelian *-subalgebra of $\mathfrak{U}$. By $\rho$ we denote the restriction of $\pi$ to $\mathfrak{B}$. Then the hull $h(J)[3]$ of $J=\operatorname{ker} \rho$ is a closed set in $\hat{\mathfrak{B}}$. Assume we can find $N+1$ distinct points $t_{1}, \ldots, t_{N+1} \in h(J)$. Then there exist disjoint open neighborhoods $U_{i}$ of $t_{i}$ in $\hat{\mathfrak{B}}$. Since $\mathfrak{B}$ is regular we can find elements $a_{i} \in \mathfrak{B}$ with $\hat{a}_{i}\left(t_{i}\right)=1$ and $\hat{a}_{i}(t)=0$ for all $t \notin U_{i}$ with $1 \leqq i \leqq N+1$. Here ${ }^{\wedge}$ denotes the Gelfand transform. Then $a_{i} a_{j}=a_{j} a_{i}=0$ for $i \neq j$ and $a_{i} \notin \operatorname{ker} \pi$. Let $c_{1}, \ldots, c_{N}$ be arbitrary in $\mathfrak{A}$, then the element $a=a_{1} c_{1} a_{2}+\ldots$ $+a_{N} c_{N} a_{N+1}$ "satisfies $a^{N+1}=0$. By assumption $a^{N}=a_{1} c_{1} a_{2}^{2} \cdots a_{N}^{2} c_{N} a_{N+1}=0$. This holds for all possible choices of $c_{i}$. Since $\pi\left(a_{N+1}\right) \neq 0$ there exists a $\xi \in H_{\pi}$ with $\pi\left(a_{v+1}\right) \xi=\eta \neq 0$. Then by assumption

$$
\pi\left(a_{1} c_{1} a_{2}^{2} c_{2} \cdots a_{N}^{2}\right) \pi\left(c_{N}\right) \eta=0 .
$$

Keeping the $c_{1}, \ldots, c_{N-1}$ fixed for a moment, this holds for all $c_{N}$. However since $\pi$ is irreducible the set $\left\{\pi\left(c_{N}\right) \eta \mid c_{N} \in \mathfrak{U}\right\}$ is dense in $H_{\pi}$. Hence $\pi\left(a_{1} c_{1} \cdots a_{N}^{2}\right)=0$ and this holds for any $(N-1)$-tuple $\left(c_{1}, \cdots, c_{N-1}\right)$. Continuation of this argument finally leads to $\pi\left(a_{1}\right)=0$, a contradiction. Hence any selfadjoint element in $\pi(\mathfrak{U})$ has at most $N$ points in its spectrum. By the lemma we get $\operatorname{dim} \pi \leqq N$.

(ii) Let $\pi$ be an irreducible representation of dimension $N$, and let $e_{i, j}$, with $1 \leqq i, j \leqq N$, be a system of matrix units in $\pi(\mathfrak{R})$. As in (i) we can find elements $a_{i} \in \mathfrak{U}$ such that $a_{i} a_{j}=a_{j} a_{i}=0$ for $i \neq j$ and $\pi\left(a_{i}\right)=e_{i, i}$. Further let $c_{i} \in \pi^{-1}\left(e_{i, i+1}\right)$ then $a=a_{1} c_{1} a_{2}+\cdots+a_{N-1} c_{N-1} a_{N}$ satisfies $a^{x}=0$ and

$$
\pi\left(a^{N-1}\right)=\pi\left(a_{1} c_{1} a_{2}^{2} \cdots a_{N-1}^{2} c_{N-1} a_{N}\right)=e_{1,1} e_{1,2} e_{2,2}^{2} \cdots e_{N, N}=e_{1, N} \neq 0 .
$$

(iii) Assume $\mathfrak{U}$ has a quasinilpotent element $x$ with $x^{N} \neq 0$.

Then there exists an irreducible representation $\pi$ with $\pi\left(x^{N}\right) \neq 0$. Since $\operatorname{dim} \pi \leqq N$ this is impossible.

For $N=1$ the theorem may be stated in slightly weaker form.

COROLlARY. Let $\mathfrak{A}$ be an $A^{*}$-algebra with normal generators $\left\{x_{i}\right\}$ such

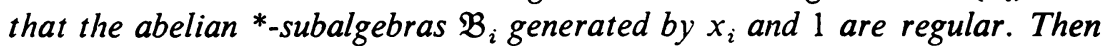
$\mathfrak{A}$ is commutative iff $\mathfrak{U}$ has no nilpotent elements.

Proof. Assume $\mathfrak{A}$ has no nilpotent elements and let $\pi$ be an irreducible representation of $\mathfrak{A}$. Then arguing as above we see that $\operatorname{Sp}\left(\pi x_{i}\right)$ consists of 
only one point. Hence $\pi\left(x_{i}\right)=\lambda_{i} 1$ and $\operatorname{dim} \pi=1$. Thus $\mathfrak{A}$ is commutative. The converse is trivial, because commutative $A^{*}$-algebras are semisimple.

The corollary applies in particular to $l^{1}$-algebras of discrete groups [1] Part (ii) of Theorem 1 can be extended to $n=\infty$ for $C^{*}$-algebras.

THEOREM 2. Let $\mathfrak{U}$ be a $C^{*}$-algebra such that there is $a \pi \in \hat{\mathfrak{U}}$ with $\operatorname{dim} \pi=\infty$. Then $\mathfrak{A}$ has an $\infty$-nilpotent element.

Proof. Again there is no loss of generality to assume that $\mathfrak{U}$ has a unit. We assume further that $\mathfrak{A}$ has no $\infty$-nilpotent elements and derive a contradiction. Let $\pi \in \hat{\mathfrak{U}}$ be an infinite dimensional representation and assume there is a $b=b^{*} \in \pi(\mathfrak{U})$ such that the spectrum of $b$ contains an interval $[\alpha, \beta]$ with $\beta>\alpha$. Using the functional calculus of $C^{*}$-algebras we find an $a=a^{*} \in \pi(\mathfrak{U})$ with Sp $a=[0,1]$. Let $c \in \mathfrak{U}$ be positive hermitean with $\pi(c)=a$ and let $f_{i}$ be the function.

$$
\begin{array}{rlrl}
f_{i}(t) & =0, & t & \leqq \frac{1}{i+1}, \\
& =\text { linear, } & \frac{1}{i+1} \leqq t \leqq \frac{3 i+1}{3 i(i+1)}, \\
& =\frac{1}{i^{2}}, & \frac{3 i+1}{3 i(i+1)} \leqq t \leqq \frac{3 i+2}{(i+1) i 3}, \\
& =\text { linear, } & \frac{3 i+2}{3 i(i+1)} \leqq t \leqq \frac{1}{i}, \\
& =0, & t & \geqq \frac{1}{i} .
\end{array}
$$

Then the elements $a_{i}=f_{i}(c)$ satisfy $a_{i} a_{j}=0$ for $i \neq j$ and $\pi\left(a_{i}\right)=f_{i}(a) \neq 0$. Now let $q=\sum_{i=1} a_{i} c_{i} a_{i+1}$ with $c_{i} \in \mathfrak{A}$ and $\left|c_{i}\right| \leqq 2$. Then

and

$$
q^{k}=\sum_{i=1}^{\infty} a_{i} c_{i} a_{i+1}^{2} \cdots c_{i+k-1} a_{i+k}
$$

$$
\left|q^{k}\right| \leqq \sum_{i=1}^{\infty} \frac{2^{k}}{i^{2}(i+1)^{3} \cdots(i+k)^{2}} \leqq \sum_{i=1}^{\infty} \frac{1}{i(i+1) \cdots(i+k)}=\frac{1}{k k !} .
$$

Hence $q$ is quasinilpotent for all possible choices of $c_{i}$. We shall show now that we can find $c_{i} \in \mathfrak{U}$ such that $\left|c_{i}\right| \leqq 2$ and such that $\pi\left(q^{n}\right) \neq 0$ for all $n$.

By construction of the $a_{i}$ there exist unit vectors $\xi_{i} \in H_{\pi}$ with $\pi\left(a_{i}\right) \xi_{i}=$ $\left(1 / i^{2}\right) \xi_{i}$. Since $\pi$ is irreducible there exist $c_{i} \in \pi(\mathfrak{Q})$ with $\left|c_{i}\right| \leqq 2$ and $\pi\left(c_{i}\right) \xi_{i+1}=\xi_{i}[2,2.8 .3]$. Let $\left\{c_{i}\right\}$ be a fixed sequence of such operators and 
assume that the corresponding $q$ satisfies $q^{k}=0$. Then

$$
\begin{aligned}
0 & =\pi\left(q^{k}\right) \xi_{k+1}=\pi\left(a_{1} \cdots a_{k}^{2}\right) \pi\left(c_{k}\right) \pi\left(a_{k+1}\right) \xi_{k+1}=\cdots \\
& =\frac{1}{(k+1)^{2}} \frac{1}{k^{4}} \cdots \frac{1}{2^{4}} \xi_{1} \neq 0 \text { a contradiction. }
\end{aligned}
$$

The case when every maximal abelian subalgebra $\mathfrak{B}$ of $\pi(\mathfrak{A})$ has a totally disconnected spectrum can be treated similarly, because dim $\pi=\infty$.

This theorem is an extension of a result of Topping [4], who showed that any antiliminal $C^{*}$-algebra possesses $\infty$-nilpotent elements. Our methods however are different and more direct. Theorem 2 shows that any $C^{*}$-algebra without $\infty$-nilpotent elements is necessarily liminal. This result is not optimal. To see this, let $\mathfrak{A}$ be a weak direct sum of liminal $C^{*}$-algebras $\mathfrak{A}_{i}, \mathfrak{A}=\Sigma^{\prime} \oplus \mathfrak{A}_{i}$, such that any $\pi \in \hat{\mathfrak{A}}_{i}$ satisfies $\operatorname{dim} \pi<$ $\infty$, but such that there exists a $\pi_{i} \in \hat{\mathfrak{A}}_{i}$ with $\operatorname{dim} \pi_{i}=p_{i}$ and $p_{i} \rightarrow \infty$. Then by Theorem 1 (ii) there exists a $p_{i}$-nilpotent element $a_{i} \in \mathfrak{U}_{i}$ with $\left|a_{i}\right|=1$. Then $a=\sum \oplus a_{i} \cdot 1 / i$ is an $\infty$-nilpotent element. This example leads immediately to our next theorem.

THEOREM 3. Let $\mathfrak{U}$ be a separable $C^{*}$-algebra without $\infty$-nilpotent elements. Then there exists a positive integer. $N$ such that any quasinilpotent element $x \in \mathfrak{A}$ satisfies $x^{N}=0$.

Proof. (a) By Theorem 2 we may assume $\mathfrak{U}$ to be liminal. Suppose further that $\mathfrak{A}$ has irreducible representations of arbitrary high but finite dimensions. Determine now as in $[2,4.4 .4]$ an open set $\mathfrak{D}_{1}^{\prime} \subset \hat{\mathfrak{A}}$ with the properties $a, b$ and $c$ of $[2,4.4 .4]$. Then there exist some positive integer $n_{1}$ and a nontrivial open subset $\mathfrak{D}_{1} \subset \mathfrak{D}_{1}^{\prime} \cap{ }_{n_{1}} \hat{\mathfrak{U}}[2,3.6 .3]$ or any nontrivial open subset of $\mathfrak{D}_{1}^{\prime}$ contains representations of arbitrary high dimensions. Similarly determine an open subset $\mathfrak{D}_{2}^{\prime}$ in $\left.\hat{\mathfrak{U}}\right|_{n_{1}} \hat{\mathfrak{U}}$. Continuing this process we either find a sequence of nonempty open sets $\mathfrak{D}_{i} \subset_{n_{i}} \hat{\mathfrak{U}}-{ }_{n_{i-1}} \hat{\mathfrak{U}}$ with $n_{i} \rightarrow \infty$ or $\hat{\mathfrak{A}}$ contains a locally compact open set $\mathfrak{D}$ such that any nontrivial open subset of $\mathfrak{D}$ contains representations of arbitrary high dimensions. In the first case let $J \subset \mathfrak{A}$ be the ideal corresponding to $\mathfrak{D}=\bigcup_{i=1}^{\infty} \mathfrak{D}_{i}$, then $J$ has the form $J=\Sigma^{\prime} \oplus J_{i}$ with $\hat{J}_{i}=\mathfrak{D}_{i}[2,1.9 .12]$. Thus by our result above $J$ contains an $\infty$-nilpotent element. In the second case determine an infinite family $\left\{\mathfrak{D}_{i}\right\}$ of nonempty disjoint open subsets of $\mathfrak{D}$. Let $J_{i}$ be the ideal corresponding to $\mathfrak{D}_{i}$. Then the proof of Theorem 2 shows that $J_{i}$ contains an $i$-nilpotent element $x_{i}$ with $\left|x_{i}\right| \leqq 2^{-i}$. Clearly $x=\sum \oplus x_{i}$ is $\infty$-nilpotent.

An amusing consequence of Theorem 3 is the following result: Let $\mathfrak{U}$ be a separable $C^{*}$-algebra; then $\hat{\mathfrak{A}}={ }_{n} \hat{\mathfrak{A}}$ iff for any singly generated subalgebra $\mathfrak{B}$ of $\mathfrak{A}$ we have $\hat{\mathfrak{B}}={ }_{n} \hat{\mathfrak{B}}$. 


\section{REFERENCES}

1. H. Behncke, Nilpotent elements in group algebras, Bull. Acad. Polon. Sci. 9 (1971), 197-198.

2. J. Dixmier, Les $C^{*}$-algèbres et leurs représentations, Cahiers Scientifiques, fasc. 29, Gauthier-Villars, Paris, 1964. MR 30 \#1404.

3. C. E. Rickart, General theory of Banach algebras, University Series in Higher Math., Van Nostrand, Princeton, N.J., 1960. MR 22 \#5903.

4. D. M. Topping, Transcendental quasi-nilpotents in operator algebras, J. Functional Analysis 2 (1968), 342-351. MR 38 \#1535.

Department of Mathematics, University of Heidelberg, Heidelberg, Federal REPUBLIC OF GeRMANY

Current address: Fakultät für Mathematik, Universität Bielefeld, 48 Bielefeld, Federal Republic of Germany 